\title{
A ketogenic diet attenuates proliferation and stemness of glioma stem-like cells by altering metabolism resulting in increased ROS production
}

\author{
CHEN-CHEN JI ${ }^{1,2^{*}}$, YI-YANG HU ${ }^{3 *}$, GUANG CHENG $^{1 *}$, LIANG LIANG $^{3 *}$, BO GAO $^{4}$, YING-PENG REN $^{1}$, \\ JIN-TAO LIU ${ }^{5}$, XIU-LI CAO ${ }^{3}$, MIN-HUA ZHENG ${ }^{3}$, SAN-ZHONG LI $^{1}$, FENG WAN $^{6}$, HUA HAN $^{1,7}$ and ZHOU FEI ${ }^{1}$ \\ ${ }^{1}$ Department of Neurosurgery, Xijing Hospital, Fourth Military Medical University, Xi'an, Shaanxi 710032; \\ ${ }^{2}$ Department of Anesthesiology, First Affiliated Hospital, Xi'an Jiaotong University, Xi'an, Shaanxi 710061; \\ ${ }^{3}$ State Key Laboratory of Cancer Biology, Department of Medical Genetics and Developmental Biology, \\ Fourth Military Medical University; ${ }^{4}$ Department of Orthopedic Surgery, Xijing Hospital, Fourth Military \\ Medical University, Xi'an, Shaanxi 710032; ${ }^{5}$ Department of Orthopedic Surgery, 413 Hospital, \\ Zhoushan, Zhejiang 316000; ${ }^{6}$ Department of Neurosurgery, Tongji Hospital, Huazhong University of \\ Science and Technology, Wuhan, Hubei 430030; ${ }^{7}$ Department of Biochemistry and Molecular Biology, \\ Fourth Military Medical University, Xi'an, Shaanxi 710032, P.R. China
}

Received May 15, 2019; Accepted October 1, 2019

DOI: 10.3892/ijo.2019.4942

\begin{abstract}
Abnormal metabolism serves a critical role in the development and progression of different types of malignancies including glioblastoma (GBM), and may therefore serve as a promising target for treatment of cancer. Preclinical studies have indicated that a ketogenic diet (KD) may exhibit beneficial effects in patients with GBM; however, the underlying mechanisms remain incompletely understood. The aim of the present study was to evaluate the effects of a $\mathrm{KD}$ on glioma stem-like cells (GSCs), by culturing patient-derived primary GSCs as well as a GSC cell line in glucose-restricted, $\beta$-hydroxybutyrate-containing medium (BHB- $\mathrm{G}^{\text {low }}$ ) which was used to mimic clinical KD treatment. GSCs cultured
\end{abstract}

Correspondence to: Professor Hua Han or Professor Zhou Fei, Department of Neurosurgery, Xijing Hospital, Fourth Military Medical University, 169 Chang-Le Xi Street, Xi'an, Shaanxi 710032, P.R. China

E-mail: huahan@fmmu.edu.cn

E-mail: feizhou@fmmu.edu.cn

${ }^{*}$ Contributed equally

Abbreviations: mTOR, mammalian target of rapamycin; Glut1, glucose transport 1; HK1/2, hexokinase1/2; PK, pyruvate kinase; PKM1/2, M1/2 isoform of pyruvate kinase; PFK, phosphofructokinase; PFKP, phosphofructokinase platelet-type; LDHA, lactate dehydrogenase A; PDH, pyruvate dehydrogenase; NAC, $\mathrm{N}$-acetylcysteine

Key words: glioblastoma, ketogenic diet, glioma stem-like cells, reactive oxygen species, glycolysis in BHB- $\mathrm{G}^{\text {low }}$ medium exhibited reduced proliferation and increased apoptosis compared with cells grown in the control medium. Furthermore, decreased expression of stem cell markers, diminished self-renewal in vitro, and reduced tumorigenic capacity in vivo, providing evidence that the stemness of GSCs was compromised. Mechanistically, culturing in $\mathrm{BHB}-\mathrm{G}^{\mathrm{low}}$ medium reduced glucose uptake and inhibited glycolysis in GSCs. Furthermore, culturing in the BHB- $\mathrm{G}^{\text {low }}$ medium resulted in morphological and functional disturbances to the mitochondria of GSCs. These metabolic changes may have reduced ATP production, promoted lactic acid accumulation, and thus, increased the production of reactive oxygen species (ROS) in GSCs. The expression levels and activation of mammalian target of rapamycin, hypoxia-inducible factor 1 and B-cell lymphoma 2 were decreased, consistent with the reduced proliferation of GSCs in BHB- $\mathrm{G}^{\text {low }}$ medium. ROS scavenging reversed the inhibitory effects of a KD on GSCs. Taken together, the results demonstrate that treatment with KD inhibited proliferation of GSCs, increased apoptosis and attenuated the stemness in GSCs by increasing ROS production.

\section{Introduction}

Aberrant metabolism in cancer cells, referred to as the Warburg effect, has been demonstrated in various types of tumor, including glioblastoma (GBM). GBM is the most common and aggressive type of brain tumor and is associated with an extremely poor prognosis in adults, with a median survival time of 9-12 months and a 5-year overall survival rate of $<5 \%(1,2)$. Similar to numerous other malignancies, GBM is a highly metabolically active malignancy, where glycolysis is the major pathway for energy metabolism (3), although both of the two major energy metabolism pathways, mitochondrial oxidative 
phosphorylation and aerobic glycolysis exist in these cancer cells (4). This metabolic pattern shift or energy metabolism reprogramming towards glycolysis provides cancer cells with not only a sufficient source of energy, but also with numerous metabolic intermediates and products that are essential for biosynthesis and various malignant behaviors (5). Although the mechanism underlying metabolic reprogramming remains incompletely understood, research has indicated the involvement of mutational and epigenetic alterations of glucose transporters (Gluts) (6,7) and key enzymes of glycolysis and associated metabolic pathways in cancer cells as well as damage to mitochondrial structure and function (8).

Metabolic pathways have received increasing attention as promising potential targets for treatment of cancer. A ketogenic diet (KD) consists of high-fat foods and appropriate proteins with only minimal carbohydrate intake, leading to physiological ketosis by lowering blood glucose levels and increasing the levels of blood ketone bodies following administration $(5,9)$. Studies have shown that a KD exhibits promising anti-tumor effects against GBM due to its induction of physiological ketosis (10-12). It is hypothesized that a KD targets the glycolytic phenotype of GBM cells and modulates oxidative stress in tumors (13). However, a complete understanding of the effects, as well as the underlying mechanisms of a KD in GBM have not been well characterized.

GBM is comprised of heterogeneous tumor cell subpopulations including glioma stem-like cells (GSCs), which are tumor cells with stem cell characteristics, including upregulated expression of stem cell markers, enhanced self-renewal and tumorigenic ability, and multi-lineage differentiation potential $(14,15)$. GSCs have been shown to be responsible for drug-resistance and tumor-recurrence in GBM (16), and research has shown that GSCs undergo a unique pattern of metabolic reprogramming that is essential for maintenance of their stemness in processes including proliferation, self-renewal, lineage specification and aging (17). Within this unique pattern, glycolysis is increased notably, suggesting that the Warburg effect may be a driving force in tumor heterogeneity $(18,19)$, and targeting of the metabolic reprogramming of GSCs may be a promising treatment for GBM (20-22). In the present study, GSCs were cultured in a ketogenic environment achieved using glucose-restricted, $\beta$-hydroxybutyrate (BHB)-containing medium (BHB- $\mathrm{G}^{\text {low }}$ ) and investigated the effects of KD treatment on GSCs as well as the underlying mechanisms. In the present study, it was hypothesized that a KD may exhibit inhibitory effects on GSC proliferation and compromise GSC stemness, possibly by disturbing metabolic balance and mitochondrial function.

\section{Materials and methods}

Cell culture. The GSC line NCH421k was kindly provided by Professor C. Herold-Mende (Ruprecht Karl University of Heidelberg, Heidelberg-Baden, Germany) (23). Normal neural stem-like cells (NSCs) were isolated from brain cortices of mice at embryonic day 14.5 as described in our previous study (24) and used as the control cells.

For primary culture, tumor tissues (Supplementary methods) were dissociated mechanically, and single-cell suspensions were cultured in serum-free DMEM/F12 medium
(Gibco; Thermo Fisher Scientific, Inc.) supplemented with $20 \mathrm{ng} / \mathrm{ml}$ epidermal growth factor, $20 \mathrm{ng} / \mathrm{ml}$ basic fibroblast growth factor (Pepro Tech, Inc.) and B-27 supplements (1:100; Gibco; Thermo Fisher Scientific, Inc.). To mimic a KD, cells were cultured in low glucose $(2.5 \mathrm{mM})$ medium (Gibco; Thermo Fisher Scientific, Inc.) supplemented with $0,1,5$ or $10 \mathrm{mM}$ BHB (Sigma-Aldrich; Merck KGaA) and termed BHB-G ${ }^{\text {low }}$ with or without $5 \mathrm{mM}$ (reactive oxygen species) ROS scavenger N-acetylcysteine (NAC) (Sigma-Aldrich; Merck KGaA).

Primary tumor spheres of NCH421k cells and patient derived GSCs were counted under a microscope on day 7 following plating. For re-plating, the primary spheres were mechanically dissociated into single-cell suspensions and re-plated at a density of $1 \times 10^{5}$ cells $/ \mathrm{ml}$ for culture as described above for another 7 days until secondary tumor spheres were observed. NCH421k cell culture, tumor spheroid formation and the re-plating assay was performed as described previously (25).

In vivo glioma model. Athymic nude mice (nu/nu), 4-6-weeks-old, were housed in a specific-pathogen-free facility. Nude mice were anesthetized with $3 \%$ isoflurane and anesthesia was maintained with $3.5 \%$ chloral hydrate, $350 \mathrm{mg} / \mathrm{kg}$. After confirming, anesthesia (lack of pain and blink reflexes), the procedure was performed. To obtain cells for in situ inoculation, $\mathrm{NCH} 421 \mathrm{k}$ cells were expanded in culture under low glucose medium with or without $10 \mathrm{mM}$ BHB for 7 days, and $5 \times 10^{5}$ viable cells were injected intracranially into mice as described previously (23). The mice were observed once every 2 days and maintained until they exhibited neurological symptoms, such as lethargy, ataxia, seizures and/or paralysis, or until their body weight was reduced by $20 \%$ compared with the initial value. A total of 8 weeks later, all mice were sacrificed. The mice brains were harvested, fixed in $4 \%$ paraformaldehyde (PFA) at room temperature (RT) overnight, and embedded in paraffin for further analyses. All animal procedures were performed in accordance with protocols approved by the Ethics Committee of Fourth Military Medical University (approval no. XJ20151231).

Hematoxylin and eosin staining. The 2-3 $\mu \mathrm{m}$ slides were deparaffinized using xylene and rehydrated with a graded ethanol series (100, 95 and 80\%). Subsequently, each slide was incubated with eosin (Thermo Fisher Scientific, Inc.) for a few seconds at RT and washed with $\mathrm{ddH}_{2} \mathrm{O}$. Next, the sections were counterstained with hematoxylin (Thermo Fisher Scientific, Inc.) at RT for a few seconds and mounted on cover slips. Brightfield microscopy was used to image the tissues using an Olympus BX51 microscope (Olympus Corporation) at $\mathrm{x} 4$ or x20 magnification.

Cell viability and proliferation. A Cell Counting Kit-8 (CCK8) assay kit (Dojindo Molecular Technologies, Inc.) was used to examine cell viability. Tumor spheres were seeded in 96-well plates $\left(2 \times 10^{4}\right.$ cells/well $)$ with medium containing $2.5 \mathrm{mM}$ glucose and 0 or $10 \mathrm{mM}$ BHB. Cells cultured in medium containing $25 \mathrm{mM}$ glucose was used as the control. For analysis of viability at the end of the culture experiments, cells were incubated with $10 \mu \mathrm{l}$ CCK8 reaction solution (per $100 \mu \mathrm{l}$ 
culture medium) at $37^{\circ} \mathrm{C}$ for $40 \mathrm{~min}$, and the optical density at $450 \mathrm{~nm}$ was measured using a Bio-Rad 680 microplate reader (Bio-Rad Laboratories, Inc.).

A 5-ethynyl-2'-deoxyuridine (EdU) assay was used to evaluate cell proliferation. Briefly, cells were incubated with $50 \mu \mathrm{M}$ EdU (Guangzhou RiboBio Co., Ltd.) in medium for $2 \mathrm{~h}$ and then fixed with 4\% PFA at room temperature for 30 min, followed by staining with Apollo ${ }^{\circledR} 567$ (Guangzhou RiboBio Co., Ltd.) at RT for 30 min. Positively stained cells were imaged using fluorescence microscopy (Olympus, BX51) at $\mathrm{x} 10$ or $\mathrm{x} 20$ magnification.

Flow cytometry. For FACS analysis, single-cell suspensions of GSCs were stained with specific antibodies (Table SI) according to the manufacturer's protocols. Flow cytometry was performed using a FACSCalibur ${ }^{\mathrm{TM}}$ flow cytometer (BD Immunocytometry Systems). FlowJo version X.0.6 (FlowJo LLC) was used for data analysis, and dead cells were excluded based on their staining with propidium iodide (PI) (BD Biosciences) at RT for $5 \mathrm{~min}$.

Annexin V-FITC staining (BD Biosciences) was performed to evaluate cell apoptosis. Briefly, single-cell suspensions of GSCs were staining with $5 \mu \mathrm{l}$ Annexin V-FITC at RT for $15 \mathrm{~min}$. After washing with binding buffer, samples were analyzed using flow cytometry as described above.

For ROS and mitochondrial staining, DCFH-DA (Beyotime Institute of Biotechnology) and MitoTacker (Invitrogen; Thermo Fisher Scientific, Inc.) were used, respectively, according to the manufacturer's protocols. Samples were analyzed using flow cytometry as described above.

Glucose transport assay. The fluorescent D-glucose analog v2-[N-(7-nitrobenz-2-oxa-1,3-diaxol-4-yl) amino]-2-deoxyglucose (2-NBDG; BioVision, Inc.) was used as a fluorescence probe for measurement of glucose transport. For evaluation of 2-NBDG uptake, $2 \times 10^{5}$ cells/well were seeded in 6-well plates and maintained in culture for 5 days. For fluorescence labeling, cells were first pre-incubated in glucose-free Krebs-Ringer bicarbonate (KRB) buffer (Beijing Solarbio Science \& Technology Co., Ltd.) for $15 \mathrm{~min}$, and subsequently in fresh KRB buffer supplemented with $50 \mu \mathrm{M} 2-\mathrm{NBDG}$ for $30 \mathrm{~min}$ at $37^{\circ} \mathrm{C}$ in a humidified incubator with $5 \% \mathrm{CO}_{2} / 95 \%$ air. Stained cells were quantitatively analyzed using flow cytometry.

Mitochondrial function. For mitochondrial membrane potential $(\Delta \Psi \mathrm{m})$ analysis, cells in culture medium were stained at RT with $10 \mu \mathrm{g} / \mathrm{ml} \mathrm{JC}-1$ (Beyotime Institute of Biotechnology) for 30 min before washing with binding buffer, according to the manufacturer's protocol. Cells were visualized using laser scanning confocal microscopy (FV-1000, Olympus Corporation) at $\mathrm{x} 10$ or $\mathrm{x} 20$ magnification.

The oxygen consumption rate (OCR) was assayed using an OCR assay kit (cat. no. 600800; MitoXpress ${ }^{\circledR}$ Xtra HS; Cayman Chemical Company) according to the manufacturer's protocol. Briefly, $5 \times 10^{4}$ cells/well were seeded into a black, clear bottom 96-well plate (Corning, Inc.) and maintained in cell culture medium for 5 days. Subsequently, MitoXpress ${ }^{\circledR}$ Xtra probe, $0.1 \mathrm{mg}$ glucose oxidase and $10 \mu \mathrm{l}$ antimycin A were added to each well, separately, and overlaid with HS mineral oil (MitoXpress ${ }^{\circledR}$ Xtra HS; Cayman Chemical Company), The signal from the cells was monitored for $100 \mathrm{~min}$ in $10 \mathrm{~min}$ intervals at $37^{\circ} \mathrm{C}$, with $360 \mathrm{~nm}$ excitation/645 $\mathrm{nm}$ emission wavelengths.

Transmission electron microscopy (TEM). For examination by TEM, cells were harvested and fixed in $2.5 \%$ glutaraldehyde (PBS, pH 7.4) at $4^{\circ} \mathrm{C}$ overnight. Post-fixation was performed using $1 \%$ buffered osmium tetroxide at room temperature for $2 \mathrm{~h}$ before dehydration through a graded series of ethanol solutions (30, 50, 90 and 100\%) and eventual embedding in Poly/Bed (Polysciences, Inc.) for $24 \mathrm{~h}$ at $60^{\circ} \mathrm{C}$. Ultra-thin sections $(50 \mathrm{~nm})$ were cut using an ultra-microtome and mounted on copper grids (200 meshes; Chemical Laboratory Equipment Co., Ltd.). After double staining of samples with uranyl acetate $20 \mathrm{~min}$ and lead citrate $10 \mathrm{~min}$ both at RT, TEM was performed using a JEM-2000EX electron microscope (JEOL Ltd.) at $x 5,000$ and $x 30,000$ magnification. The size of the mitochondria were calculated and analyzed using the following formula: Mitochondrial size $=4 / 3 \mathrm{x} \pi \mathrm{x}(\mathrm{axb})$; where $\mathrm{a}$ is the long axis radius and $b$ is the short axis radius. For each sample, three randomly chosen fields of view were used, and three independent experimental repeats were performed.

Reverse transcription-quantitative (RT-q)PCR. From the different groups of cultured GSCs, total RNA was extracted using TRIzol ${ }^{\circledR}$ reagent (Invitrogen; Thermo Fisher Scientific, Inc.). RT was performed using PrimeScript RT Master mix (Takara Bio, Inc.), and qPCR was performed using SYBR Select Master mix (Takara Bio, Inc.) on an ABI Prism 7500 Real-Time PCR system (version 2.0.4; Applied Biosystems; Thermo Fisher Scientific, Inc.), as described previously (25). GAPDH was used as the internal control. All RT-qPCR experiments were repeated at least three times, and the results are presented as a fold difference in the mRNA levels. The sequences of the primers used were: Sox 2 forward, 5'-ACACCAATCCCA TCCACACT-3' and reverse, 5'-GCAAACTTCCTGCAAAGC TC-3'; Nanog Sox 2 forward, 5'-CAGATGCAAGAACTCTCC A-3' and Sox2 forward, 5'-GTAGGAAGAGTAAAGGCTG-3'; Oct4 forward, 5'-ACACCA ATCCCATCCACACT-3' and reverse, Oct4 forward, 5'-ACACCAATCCCATCCACACT-3' and reverse, 5'-GCAAACTTCCTGCAAAGCTC-3'; and GAPDH forward, 5'-GCACCGTCAAGGCTGAGAAC-3' and reverse 5'-TGGTGAAGACGCCAGTGGA-3'.

Western blotting. Whole cell lysates were obtained from cultured GSCs using RIPA lysis buffer (Beyotime Institute of Biotechnology) supplemented with complete protease inhibitor cocktail (Roche Diagnostics) at $4^{\circ} \mathrm{C}$ for $30 \mathrm{~min}$. A bicinchoninic acid protein assay kit (Thermo Fisher Scientific, Inc.) was used to measure total protein concentration. Protein samples $(20 \mu \mathrm{g})$ were loaded on $10 \%$ SDS gels and resolved using SDS-PAGE. After resolving, proteins were transferred to PVDF membranes and probed with the primary at $4^{\circ} \mathrm{C}$ for $12 \mathrm{~h}$ and subsequently with secondary antibodies at RT for $2 \mathrm{~h}$ (Table SI). Signals were visualized using an enhanced chemiluminescence detection system (GE Healthcare). Protein expression was analyzed using ImageJ version 1.45 (National Institutes of Health) and normalized to $\beta$-actin.

Biochemical analyses. To measure ATP production, GSCs were seeded in 96-well plates at a density of $2 \times 10^{4}$ cells/well 

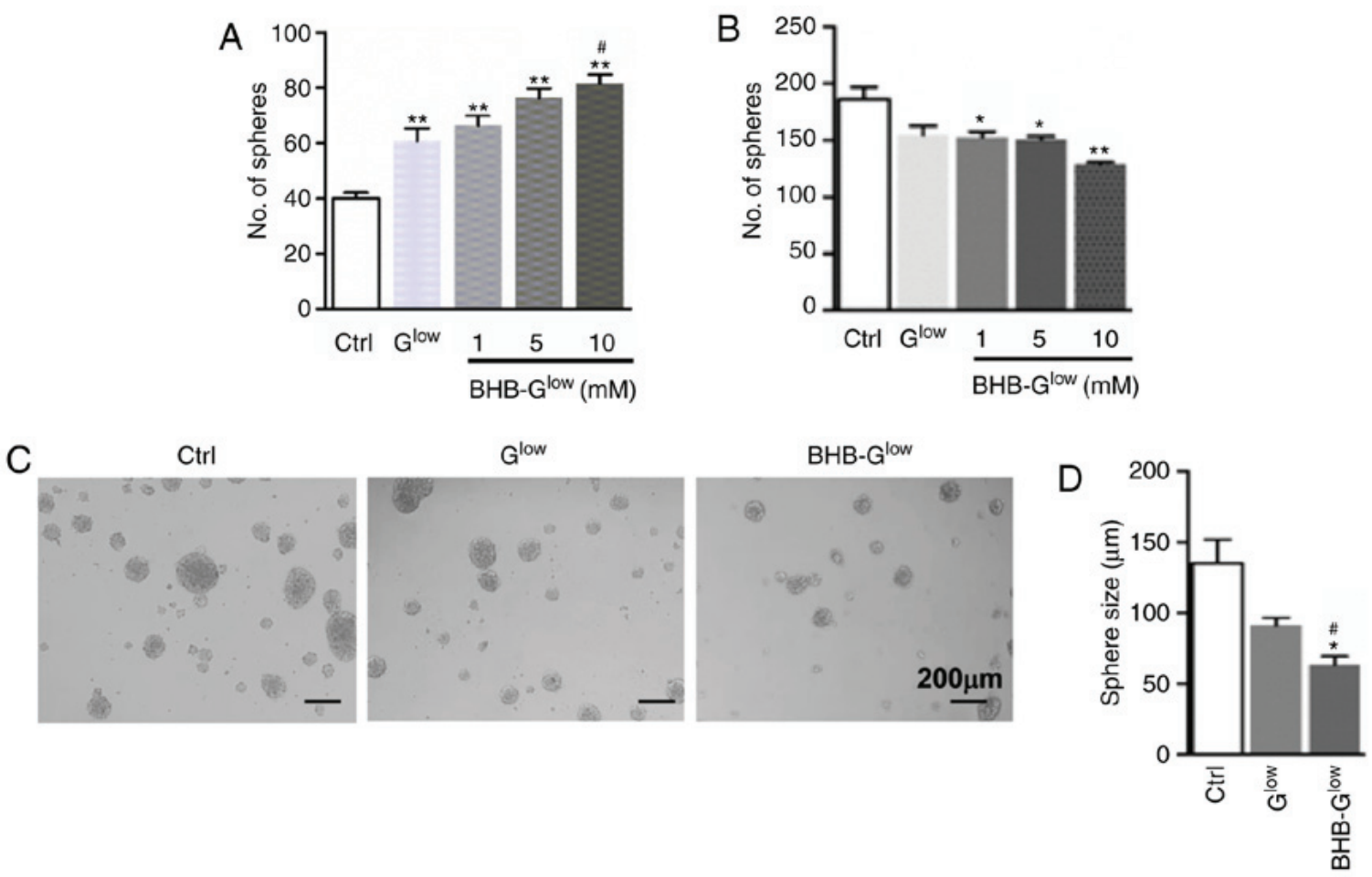

Figure 1. Effect of BHB on normal NSCs and GSCs. (A) NSCs derived from embryonic day 14.5 mouse embryos were cultured in Ctrl, $\mathrm{G}^{\mathrm{low}}$, or BHB-G ${ }^{\text {low }}$ containing either 1,5 or $10 \mathrm{mM}$ of BHB for 7 days. Neurospheres were counted under a microscope. (B) A patient-derived GSC line NCH421k was cultured in Ctrl, $\mathrm{G}^{\text {low }}$, or BHB- $\mathrm{G}^{\text {low }}$ containing either 1,5 or $10 \mathrm{mM}$ of BHB for 7 days. Tumor spheres were counted and compared. (C and D) NCH421k cells were cultured as in (B) with $10 \mathrm{mM}$ BHB for 7 days. (C) Tumor spheres were imaged using a microscope. Magnification, x10. Scale bar, $200 \mu \mathrm{m}$. Diameters of tumor spheres formed in the different growth media (D). Data are presented as the mean \pm standard deviation of three repeats. ${ }^{*} \mathrm{P}<0.05,{ }^{* *} \mathrm{P}<0.01$ vs. Ctrl; ${ }^{*} \mathrm{P}<0.05$ vs. $\mathrm{G}^{\text {low }}$. BHB, $\beta$-hydroxybutyrate; NSC, neural stem-like cell; GSC, glioma stem-like cell; Ctrl, control medium with $25 \mathrm{mM}$ glucose; $\mathrm{G}^{\text {low }}$, medium with $2.5 \mathrm{mM}$ glucose; $B H B-G^{\text {low }}, G^{\text {low }}+$ BHB.

for treatment with various concentrations of BHB. After treatment, the ATP content of the GSCs was measured using a CellTiter-Glo ${ }^{\circledast}$ assay kit (cat. no. G7570; Promega Corporation) according to the manufacturer's protocol.

To evaluate metabolic enzyme activity in cultured GSCs, cells were lysed in isolation buffers provided in the respective assay kits for phosphofructokinase (PFK), pyruvate kinase (PK) and lactate dehydrogenase (LDH) activity (all from Nanjing Jiancheng Bioengineering Institute), and the kits were used according to the manufacturer's protocols. For each enzyme, total enzyme activity was normalized to the concentration of the respective protein in the cell lysate. In addition, lactate production by GSCs was determined using a lactic acid assay kit (Nanjing Jiancheng Bioengineering Institute).

Statistical analysis. Data are expressed as the mean \pm standard deviation. GraphPad Prism version 5.0 (GraphPad Software, Inc.) was used for all data analysis. Statistical analysis was performed using repeated measures ANOVA with a post hoc Tukey's test. Data between two groups were compared using a two-sample t-test. $\mathrm{P}<0.05$ was considered to indicate a statistically significant difference.

\section{Results}

BHB inhibits the proliferation of GSCs but not normal NSCs in vitro. To investigate the effect of KD treatment on GSCs, BHB- $\mathrm{G}^{\text {low }}$ culture medium which contained a low glucose concentration $(2.5 \mathrm{mM}$, in contrast to $25 \mathrm{mM}$ in normal medium) as well as different concentrations of BHB, the major component of ketone bodies was used. As a control experiment, NSCs derived from E14.5 mouse embryos were cultured in normal medium (Ctrl), low glucose medium $\left(\mathrm{G}^{\text {low }}\right)$ or BHB- $\mathrm{G}^{\text {low }}$. The number of spheres formed were increased in NSCs grown in $\mathrm{G}^{\text {low. }}$. In addition, as the BHB concentration was increased, the number of NSC spheres formed were increased (Fig. 1A). These results suggest that BHB- $\mathrm{G}^{\text {low }}$ could support the growth of normal NSCs. NCH421k cells and patient-derived GSCs, both of which expressed the stemness markers CD133 and Nestin (Fig. S1) were cultured in $\mathrm{Ctrl}, \mathrm{G}^{\text {low }}$, or BHB- $\mathrm{G}^{\text {low }}$ medium with different concentrations of BHB for 7 days. In contrast to the NSCs, the number and size of NCH421k GSC spheres were inversely associated with $\mathrm{BHB}$ concentration, decreasing significantly as the BHB concentration was increased (Fig. 1B-D). Furthermore, primary GSCs from glioma biopsies (Supplementary file; Table SII), which stained positively for Nestin and CD133 (Fig. S1B and D), were cultured in the three different mediums. Consistent with the results obtained for the NCH421k cells, the primary GSCs formed significantly fewer and smaller tumor spheres in BHB- $\mathrm{G}^{\text {low }}$ medium containing $10 \mathrm{mM}$ BHB compared with $\mathrm{G}^{\text {low }}$ medium (Fig. S2A-C). These results suggest that BHB significantly inhibited the growth of GSCs under low glucose conditions.

BHB inhibits GSC proliferation and promotes GSC apoptosis. The effects of KD treatment on the proliferation and apoptosis 

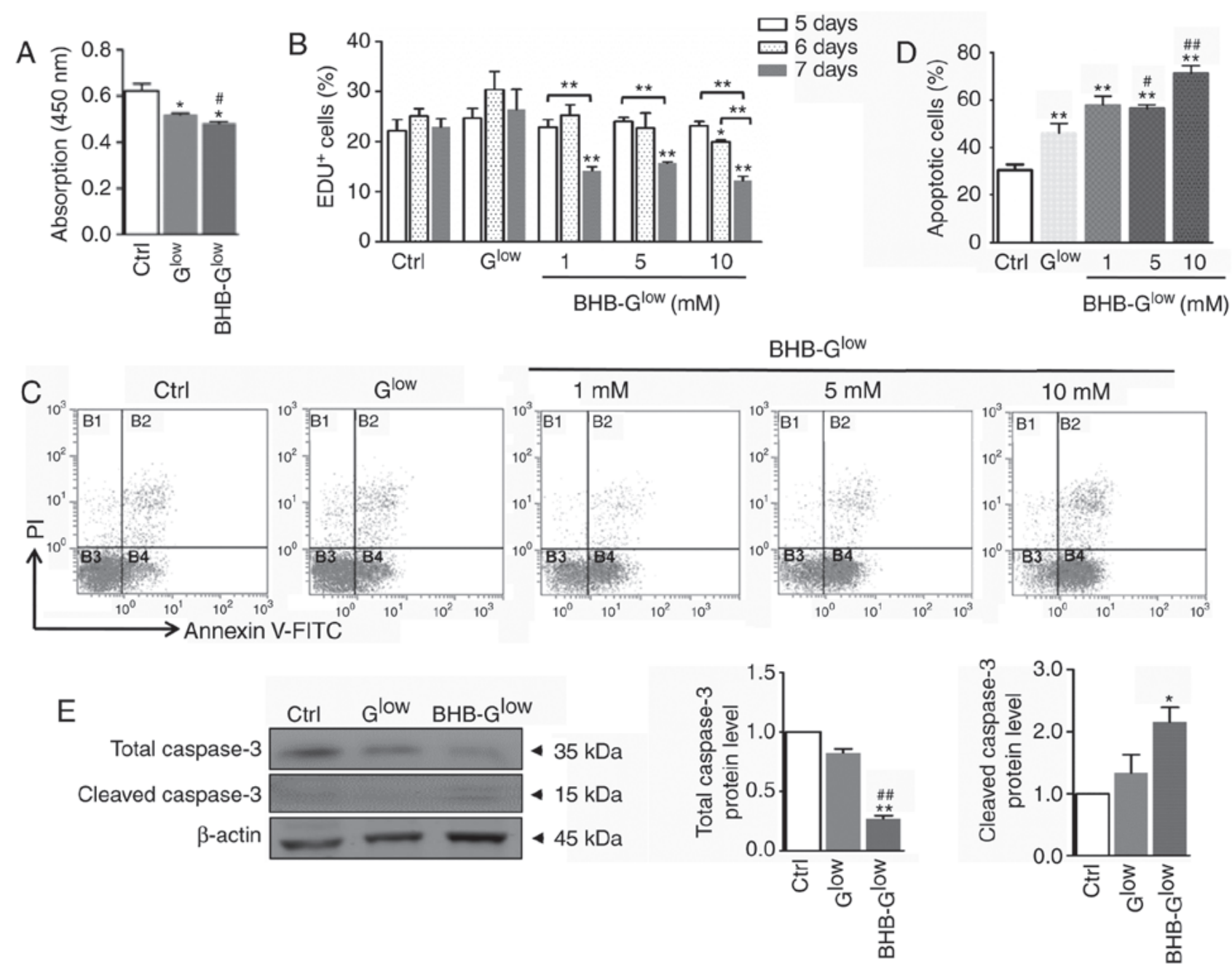

Figure 2. BHB inhibits proliferation of GSCs and promotes GSC apoptosis. (A) Cell viability was determined using Cell Counting Kit-8 analysis. (B) NCH421k cells were cultured in Ctrl, $\mathrm{G}^{\text {low }}$, or BHB- $\mathrm{G}^{\text {low }}$ medium with 1,5 or $10 \mathrm{mM}$, for either 5,6 or 7 days. Cell proliferation was determined using an EdU assay. (C) Apoptosis was determined using Annexin V staining followed by FACS analysis and (D) the results were quantitatively compared. (E) Expression levels of total-caspase 3 and cleaved-caspase 3 were determined by western blot analysis (E). Data are presented as the mean \pm standard deviation of three repeats. ${ }^{*} \mathrm{P}<0.05,{ }^{* *} \mathrm{P}<0.01$ vs. Ctrl; ${ }^{\#} \mathrm{P}<0.05,{ }^{\# \#} \mathrm{P}<0.01$ vs. G ${ }^{\text {low }}$. GSC, glioma stem-like cell; EdU, 5-ethynyl-2'-deoxyuridine; BHB, $\beta$-hydroxybutyrate; Ctrl, control medium with $25 \mathrm{mM}$ glucose; $\mathrm{G}^{\text {low }}$, medium with $2.5 \mathrm{mM}$ glucose; $B H B-\mathrm{G}^{\text {low }}, \mathrm{G}^{\text {low }}+\mathrm{BHB}$.

of GSCs was next assessed. Consistent with the reduction in GSC sphere growth, culture medium containing $10 \mathrm{mM} \mathrm{BHB}$ resulted in significantly reduced viability of $\mathrm{NCH} 421 \mathrm{k}$ cells compared with the control (Fig. 2A). Furthermore, the results of the EdU incorporation assay indicated that BHB inhibited the proliferation of primary GSCs compared with cells grown in the control medium (Fig. S2D-E). In addition, there was a significant time-dependent decrease in the percentage of $\mathrm{EdU}^{+}$ GSCs in the NCH421k cells (Fig. 2B). Annexin V staining of GSCs cultured in BHB-G ${ }^{\text {low }}$ revealed that $\mathrm{BHB}$ treatment significantly induced apoptosis in $\mathrm{NCH} 421 \mathrm{k}$ cells compared with cells grown in the control medium (Fig. 2C and D). Furthermore, cleaved-caspase 3 expression was significantly increased in the GSCs cultured in BHB- $\mathrm{G}^{\text {low }}$ compared with the control group (Fig. 2E). Together, these data suggest that BHB inhibited GSC proliferation whilst increasing GSC apoptosis under glucose deprivation.

BHB attenuates the stemness of GSCs. The effect of BHB on the stemness of GSCs was assessed. As shown in Fig. 3A-C,
BHB treatment reduced the number and size of secondary tumor spheres. The expression of the stemness marker, CD133 was determined following BHB treatment using FACS. The results showed that $\mathrm{BHB}-\mathrm{G}^{\text {low }}$ significantly decreased CD133 expression compared with the cells grown in the control medium (Fig. 3D). In addition, the expression of stemness regulators, Nanog, Oct4 and Sox2, were assessed following BHB treatment using RT-qPCR and western blotting. The results showed that $\mathrm{BHB}$ significantly decreased Nanog, Oct4 and Sox 2 expression at the mRNA level, and reduced Sox2 protein expression in NCH421k cells under glucose deprivation (Fig. 3E and F).

To investigate the effects of BHB on GSC behavior in vivo, $\mathrm{NCH} 421 \mathrm{k}$-derived secondary spheres were cultured in Ctrl, $\mathrm{G}^{\text {low }}$ or $10 \mathrm{mM}$ BHB-G ${ }^{\text {low }}$ medium and transplanted into nude mice using an orthotopic xenograft model (Fig. S2F). The tumorigenic capacity of $\mathrm{NCH} 421 \mathrm{k}$ cells cultured in $\mathrm{BHB}-\mathrm{G}^{\text {low }}$ medium was reduced in vivo (4, 2 and 1 total tumors formed in the $\mathrm{Ctrl}, \mathrm{G}^{\text {low }}$ and $10 \mathrm{mM}$ BHB-G $\mathrm{G}^{\text {low }}$ groups, respectively; $\mathrm{n}=4$ per group, data not shown). In addition, hematoxylin and 


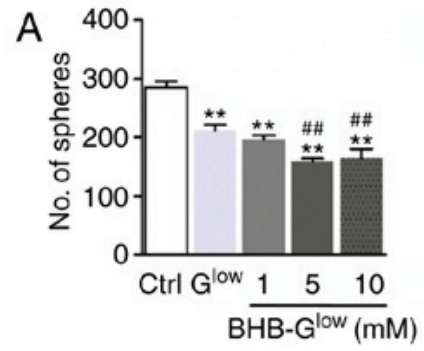

D

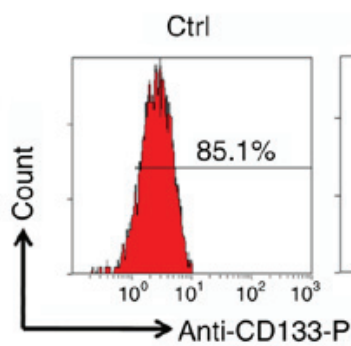

E

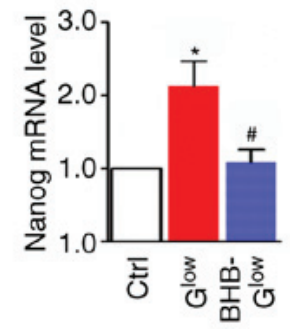

F
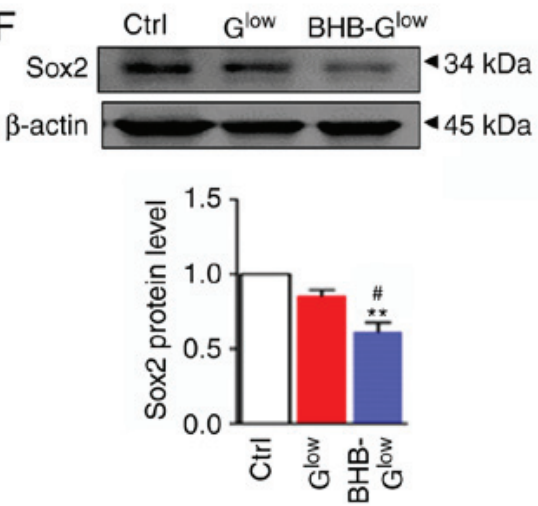

B

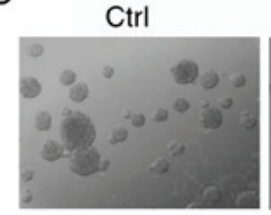

Glow

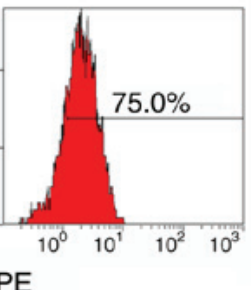

Glow
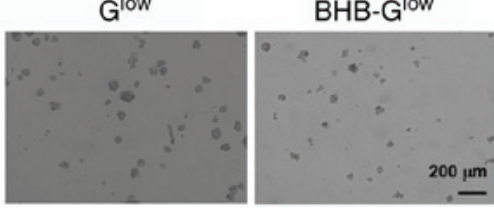

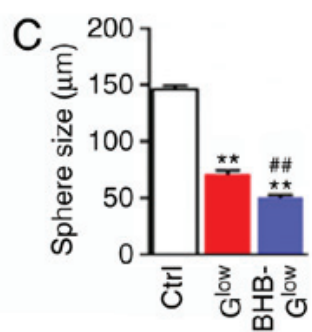

BHB-Glow
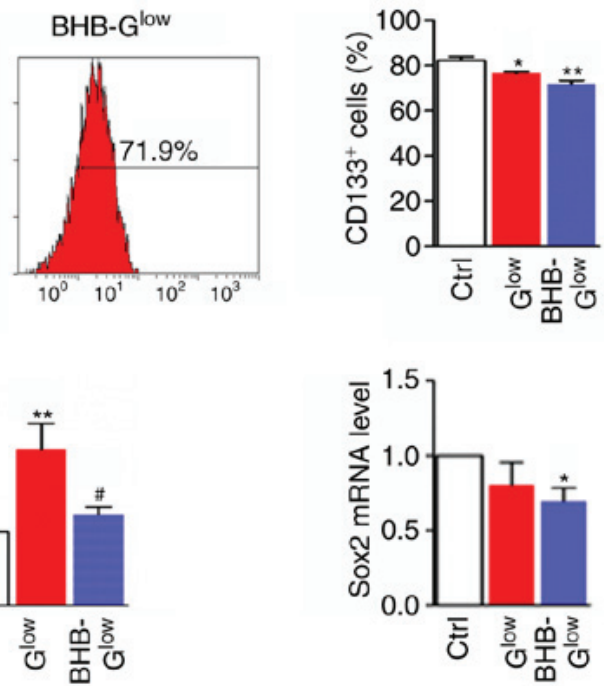
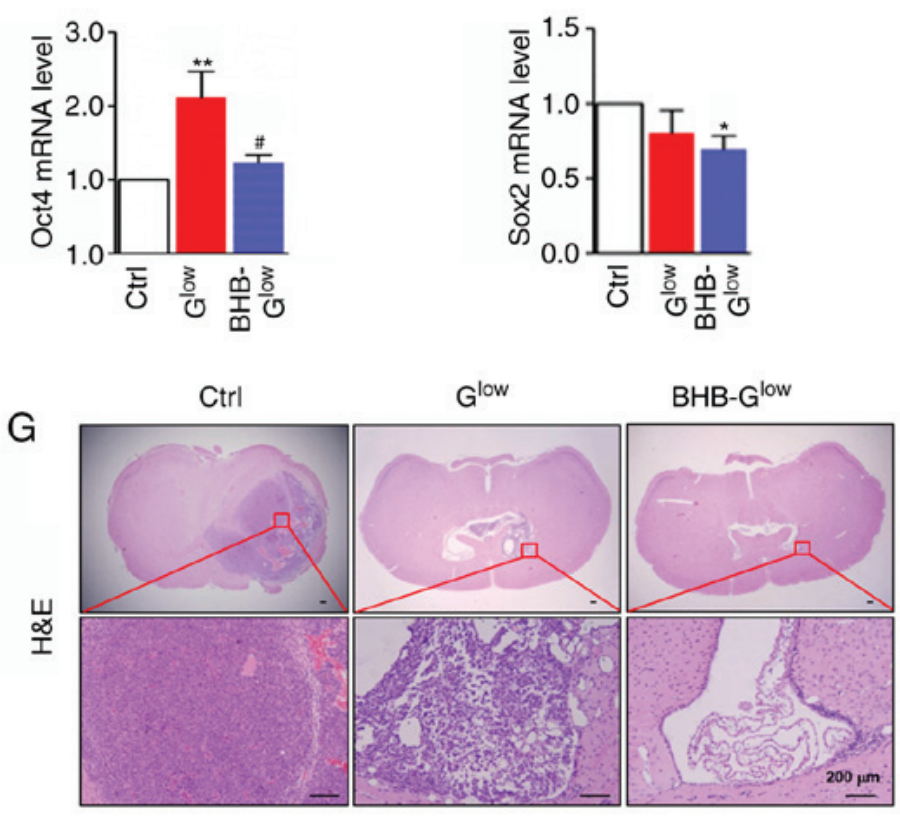

Figure 3. BHB attenuates stemness of GSCs. Secondary tumor spheres formed by NCH421k cells were (A) counted, (B) imaged and (C) the diameter of tumor spheres were compared. Scale bar, $200 \mu \mathrm{m}$. (D) Secondary tumor spheres formed in Ctrl, $\mathrm{G}^{\text {low }}$ and $10 \mathrm{mM}$ BHB-G ${ }^{\text {low }}$ culture medium were dispersed and analyzed by flow cytometry for CD133 and CD133+ cells were quantitatively compared. (E) mRNA expression levels of stemness regulators Nanog, Oct 4 and Sox2. (F) Western blot and densitometry analysis of Sox 2 expression in the secondary tumor spheres grown in Ctrl, $\mathrm{G}^{\text {low }}$ and $10 \mathrm{mM} B \mathrm{BB}-\mathrm{G}^{\text {low }}$ medium ( $\mathrm{n}=3$ ). (G) Secondary tumor spheres grown in $\mathrm{Ctrl}, \mathrm{G}^{\text {low }}$ and $10 \mathrm{mM}$ BHB-G ${ }^{\text {low }}$ culture medium were dispersed and orthotopically implanted into the right side of the brain of nude mice $(\mathrm{n}=4)$. Tumor tissues were examined by hematoxylin and eosin staining. Scale bar, $200 \mu \mathrm{m}$. Data are presented as the mean \pm standard deviation. ${ }^{*} \mathrm{P}<0.05,{ }^{* *} \mathrm{P}<0.01$ vs. Ctrl; ${ }^{\#} \mathrm{P}<0.05$, ${ }^{\# \#} \mathrm{P}<0.01$ vs. $\mathrm{G}^{\text {low }}$. GSC, glioma stem-like cell; BHB, $\beta$-hydroxybutyrate; Ctrl, control medium with 25 mM glucose; $\mathrm{G}^{\text {low }}$, medium with $2.5 \mathrm{mM}$ glucose; $\mathrm{BHB}-\mathrm{G}^{\text {low }}, \mathrm{G}^{\text {low }}+\mathrm{BHB}$.

eosin staining revealed that the volume of the tumor formed by the BHB-treated GSCs was smaller compared with the tumors formed from cells grown in the $\mathrm{Ctrl}$ and $\mathrm{G}^{\text {low }}$ media (Fig. 3G). These data suggest that BHB attenuated the stemness of GSCs under glucose deprivation.

BHB treatment inhibits glycolysis. Tumor cells undergo metabolic reprogramming to adopt a metabolic pattern of aerobic glycolysis, a process known as the Warburg effect (5). BHB is oxidized in the mitochondria, and therefore, to investigate the mechanisms of its effects on GSCs, energy metabolism within $\mathrm{NCH} 421 \mathrm{k}$ cells treated with $\mathrm{BHB}$ was assessed. An upregulation of Glut1 and several glycolysis-associated enzymes including HK2, PKM2 and LDHA in tumor tissues was observed compared with the paratumoral 'normal' brain tissues (Fig. S3A-B). Expression of pyruvate dehydrogenase (PDH), which is associated with the tricarboxylic acid cycle flux, was decreased significantly. The expression of glycolysis-associated enzymes in GSCs cultured in normal, $\mathrm{G}^{\text {low }}$ or BHB-G ${ }^{\text {low }}$ medium was assessed by western blotting. The results showed that $\mathrm{BHB}$ significantly reduced the expression of Glut1, HK1/2, PKM2, LDHA and PDH, but the protein levels of PFKP were not altered (Fig. 4A and B). The activity of PFK, PK and LDH were assessed, and it was demonstrated 

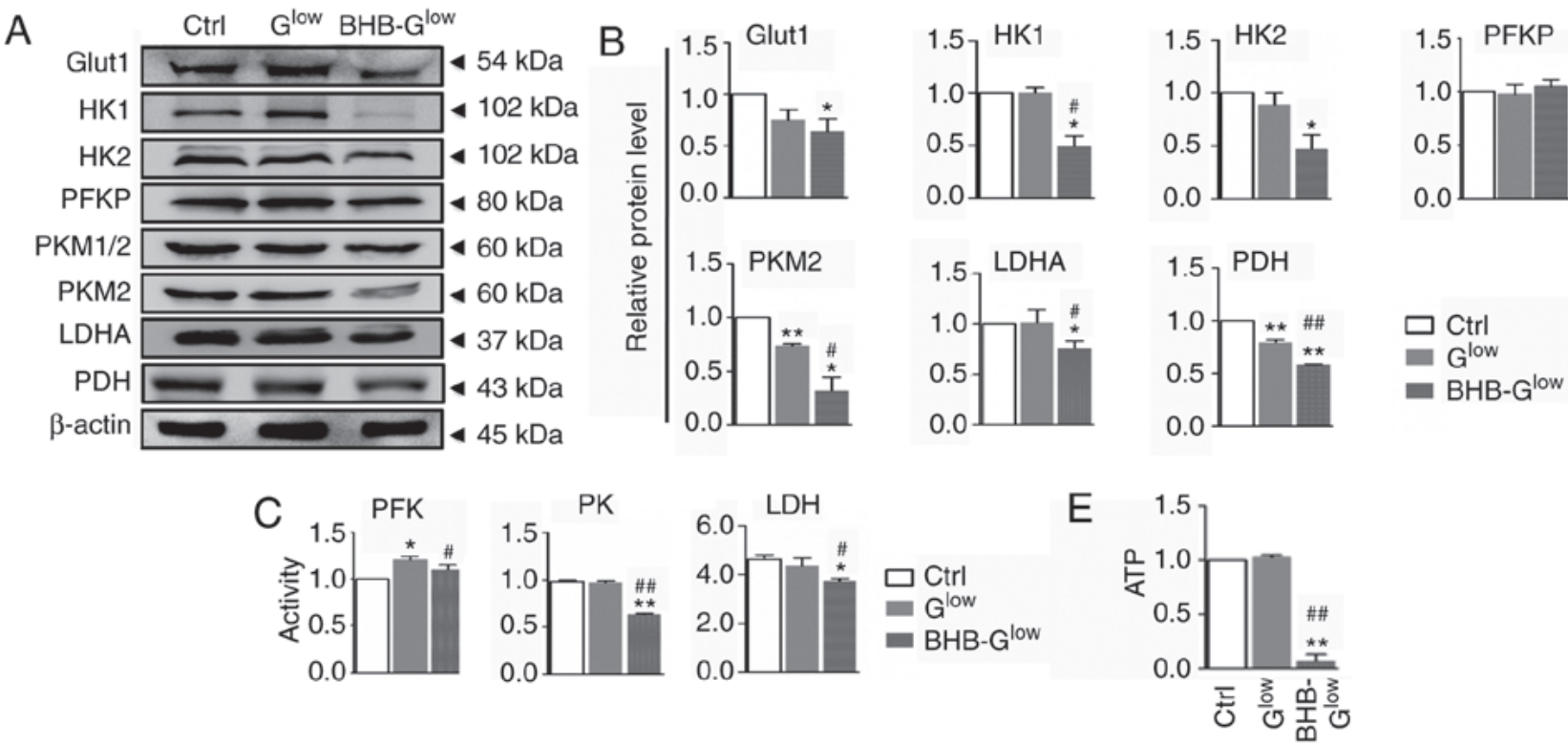

D

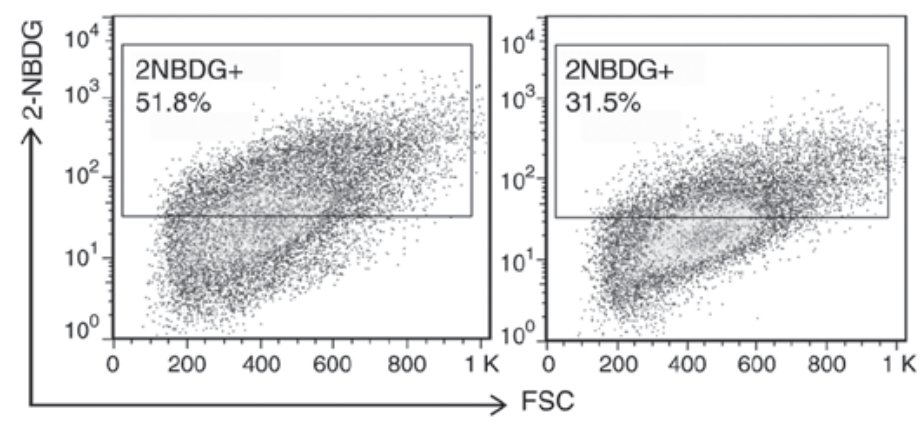

$\mathrm{G}^{\text {low }}$

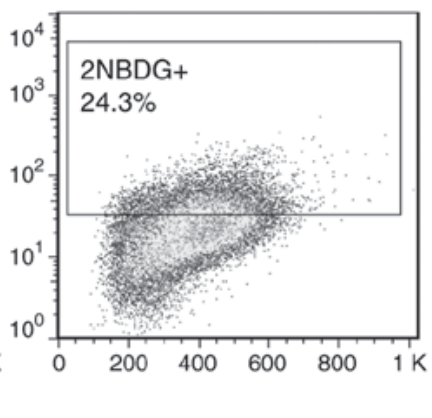

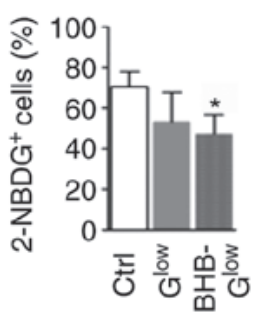

Figure 4. BHB inhibits glycolysis in GSCs. Protein expression levels of Glut1 and glycolysis-related enzymes, HK1 and 2, PFKP, PKM1/2, PKM2, LDHA and $\mathrm{PDH}$, were determined by (A) western blotting and (B) quantitatively compared using $\beta$-actin expression for normalization. (C) Activity of PFK, PK and LDH were determined. (D) Glucose transport was determined by 2-NBDG staining followed by FACS and quantitatively compared. (E) ATP production of cells grown in the different culture mediums were compared. Data are presented as the mean \pm standard deviation of three repeats. ${ }^{*} \mathrm{P}<0.05,{ }^{* *} \mathrm{P}<0.01$ vs. Ctrl; ${ }^{\#} \mathrm{P}<0.05,{ }^{\# /} \mathrm{P}<0.01$ vs. $\mathrm{G}^{\text {low }} ; \mathrm{Ctrl}$, control medium with $25 \mathrm{mM}$ glucose; $\mathrm{G}^{\text {low }}$, medium with $2.5 \mathrm{mM}$ glucose; BHB-G ${ }^{\text {low }}, \mathrm{G}^{\text {low }}+\mathrm{BHB}$. GSC, glioma stem-like cell; BHB, $\beta$-hydroxybutyrate; Ctrl, control medium with 25 mM glucose; $\mathrm{G}^{\text {low }}$, medium with 2.5 mM glucose; BHB-G ${ }^{\text {low }}, \mathrm{G}^{\text {low }}+\mathrm{BHB}$; Glut1, glucose transport 1; HK1/2, hexokinase1/2; PK, pyruvate kinase; PKM1/2, M1/2 isoform of pyruvate kinase; PFKP, phosphofructokinase platelet-type; LDHA, lactate dehydrogenase A; 2-NBDG, v2-[N-(7-nitrobenz-2-oxa-1,3-diaxol-4-yl) amino]-2-deoxyglucose; FSC, forward scatter.

that BHB treatment suppressed the activity of these glycolysis enzymes under glucose deprivation (Fig. 4C). Furthermore, transmembrane glucose transport was assessed using a fluorescent derivative of glucose 2-NBDG (26). Cells cultured in BHB-G ${ }^{\text {low }}$ medium reduced glucose uptake in NCH421k cells as evidenced by the decreased percentage of these cells that exhibited 2-NBDG fluorescence (Fig. 4D). Consistently, BHB treatment significantly reduced ATP production in $\mathrm{NCH} 421 \mathrm{k}$ cells under glucose deprivation (Fig. 4E). Taken together, these results suggest that $\mathrm{BHB}$ attenuated glycolysis, which likely resulted in an energy crisis in the GSCs.

BHB causes mitochondrial damage in GSCs. The Warburg effect is often associated with mitochondrial damage $(27,28)$. Therefore, mitochondrial function and morphology was assessed in NCH421k cells cultured in BHB-G ${ }^{\text {low }}$ medium. BHB treatment increased the OCR and lactate production in GSCs, suggesting impaired oxidative phosphorylation (Fig. 5A and B). TEM revealed decreased mitochondrial size in $\mathrm{NCH} 421 \mathrm{k}$ cells treated with BHB under glucose deprivation (Fig. 5C). Furthermore, the alterations in the mitochondrial transmembrane potential was determined using JC-1 staining. The results showed that $\mathrm{BHB}$ treatment induced depolarization of the mitochondrial membrane potential in GSCs under glucose deprivation (Fig. 5D). These results suggested that BHB impaired mitochondrial structure and function in glucose deprived GSCs.

BHB treatment increases ROS production in GSCs. Mitochondrial dysfunction is often associated with increased ROS production. ROS production was assessed in $\mathrm{NCH} 421 \mathrm{k}$ cells cultured in the different media conditions. Culturing cells in $\mathrm{G}^{\text {low }}$ and $\mathrm{BHB}-\mathrm{G}^{\text {low }}$ medium resulted in significantly increased ROS production in GSCs (Fig. 6A). Activity of the anti-oxidative enzymes catalase (CAT) and superoxide dismutase (SOD), as well as the concentration of reduced-glutathione (GSH) and oxidized glutathione (GSSG) were measured. The results showed that BHB significantly 

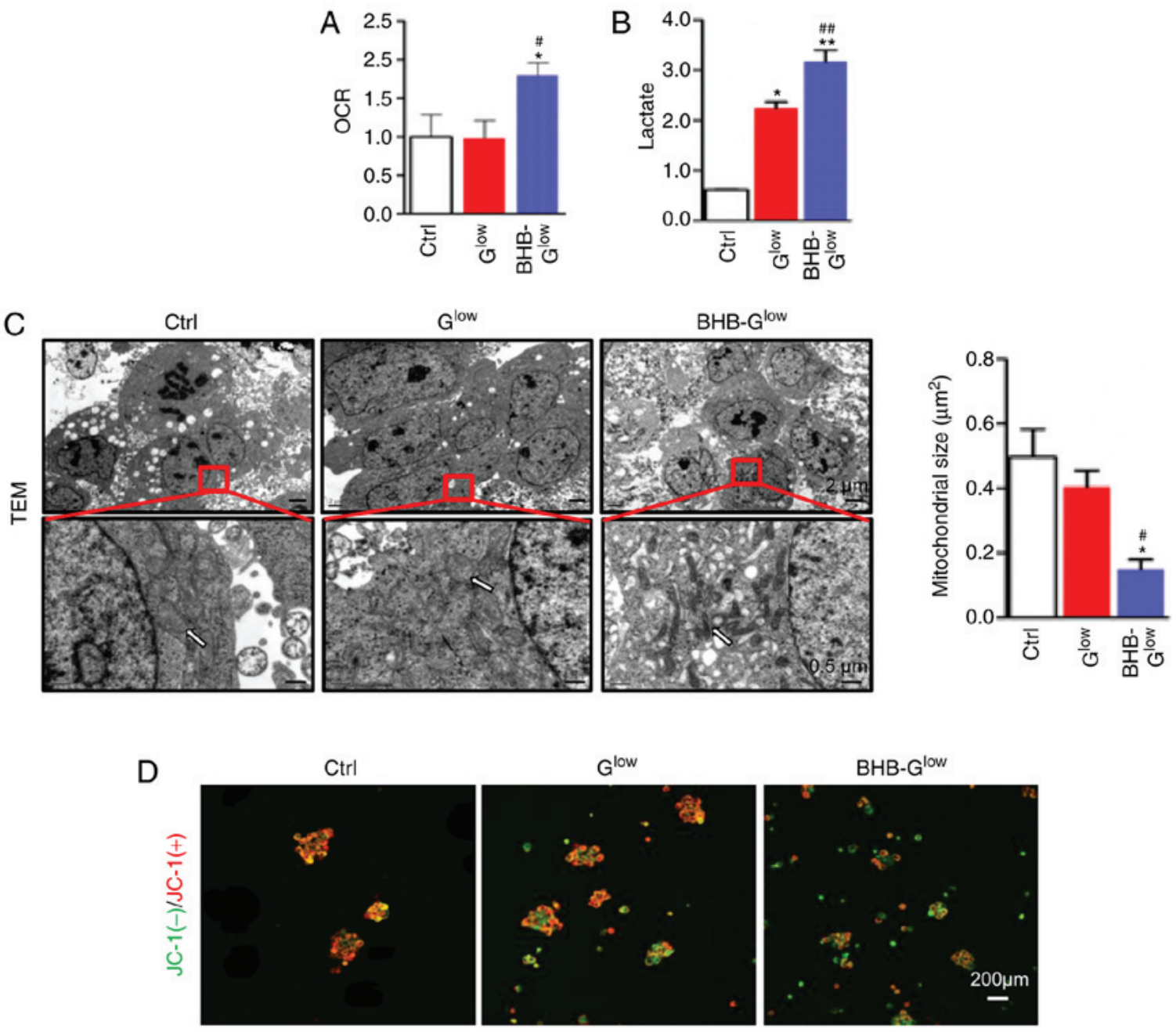

Figure 5. BHB induces mitochondrial damage in GSCs. (A) OCR (fold change relative to the control) and (B) lactate production of NCH421k cells cultured in $\mathrm{Ctrl}, \mathrm{G}^{\text {low }}$ or $10 \mathrm{mM}$ BHB-G ${ }^{\text {low }}$ medium for 5 days $(\mathrm{n}=3$ ). (C) Morphology of mitochondria (white arrows) was observed by TEM and (B) mitochondrial size was quantified. Scale bar, $2 \mu \mathrm{m}$ (top) or $0.5 \mu \mathrm{m}$ (bottom). (D) Mitochondrial membrane potential was analyzed using JC-1 staining and fluorescence microscopy. Red, JC-1(+); green, JC-1(-). Scale bar, $200 \mu \mathrm{m}$. Data are presented as the mean \pm standard deviation. ${ }^{*} \mathrm{P}<0.05,{ }^{* *} \mathrm{P}<0.01$ vs. Ctrl; ${ }^{\#} \mathrm{P}<0.05$, ${ }^{\# \prime} \mathrm{P}<0.01$ vs. Glow GSC, glioma stem-like cell; BHB, $\beta$-hydroxybutyrate; Ctrl, control medium with $25 \mathrm{mM}$ glucose; $\mathrm{G}^{\text {low }}$, medium with $2.5 \mathrm{mM}$ glucose; $B \mathrm{BH}^{\mathrm{G}} \mathrm{G}^{\text {low }}, \mathrm{G}^{\text {low }}+\mathrm{BHB}$; TEM, transmission electron microscopy.

decreased the activity of CAT and SOD and the concentration of GSH, in addition to increasing the concentration of GSSG (Fig. S4A). Furthermore, staining with an ROS probe, DCFH-DA, and labeling mitochondria with MitoTracker followed by FACS analysis indicated that the increased ROS levels originated from the mitochondria. In addition, BHB increased mitochondrial-generated ROS levels, compared with $\mathrm{G}^{\text {low }}$ (Fig. 6B). NCH421k cells could be separated into two subpopulations using MitoTracker with respect to mitochondrial mass. The number of cells with a larger mitochondrial mass, which were recognized as GSCs (29) and appeared to be major contributors of ROS, were increased in the $\mathrm{G}^{\text {low }}$ and BHB-G $\mathrm{G}^{\text {low }}$ groups (Fig. 6B). Double staining with the ROS probe and CD133 antibody indicated that although the low glucose conditions increased ROS production in $\mathrm{CD} 133^{+}$ GSCs, BHB treatment further increased ROS production in $\mathrm{CD}_{133^{+}}$GSCs compared with $\mathrm{G}^{\text {low }}$ (Fig. 6C). The expression levels of mTOR, hypoxia-inducible factor $1 \alpha$ (HIF-1 $\alpha)$ and B-cell lymphoma 2 (Bcl2), which are all associated with cell metabolism and survival, were reduced significantly in
NCH421k cells following treatment with BHB-G ${ }^{\text {low }}$ (Fig. 6D). Together, these results suggested that BHB promoted ROS production in GSCs, which may serve a critical role in the BHB-induced attenuation of GSC stemness and proliferation as well as in the increase in GSC apoptosis.

ROS scavenging reverses the effects of BHB on GSCs. To further demonstrate the role of ROS in BHB-mediated inhibition of GSC proliferation, the effects of the ROS scavenger $\mathrm{N}$-acetylcysteine (NAC) were determined on GSCs treated with BHB. The results showed that NAC reversed the inhibition of BHB on an unidentified anti-oxidative signaling pathway by increasing the activity of SOD, CAT and the concentrations of GSH, as well as decreasing the concentration of GSSG (Fig. S4B). Additionally, NAC increased tumor sphere formation in NCH421k cells at concentrations $\leq 25 \mathrm{mM}$ (Fig. S3C). In the presence of NAC, the number and size of tumor spheres that were formed from by $\mathrm{NCH} 421 \mathrm{k}$ cells treated with BHB, as well as cell viability within the spheres, was increased (Fig. 7A-C). In addition, western blotting revealed that NAC 
A

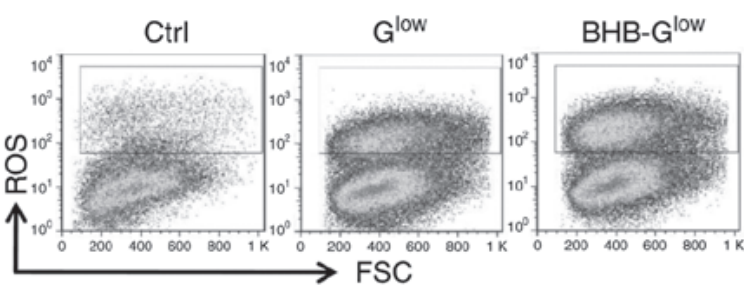

B
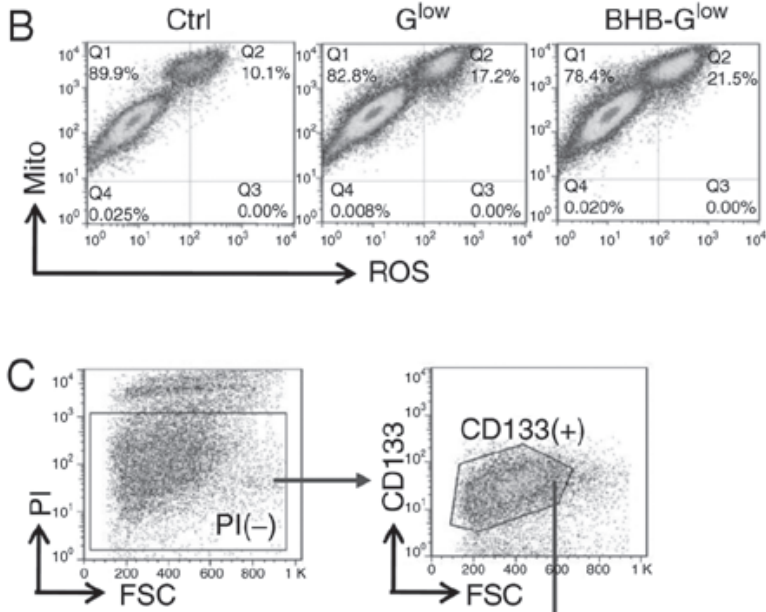

Ctrl
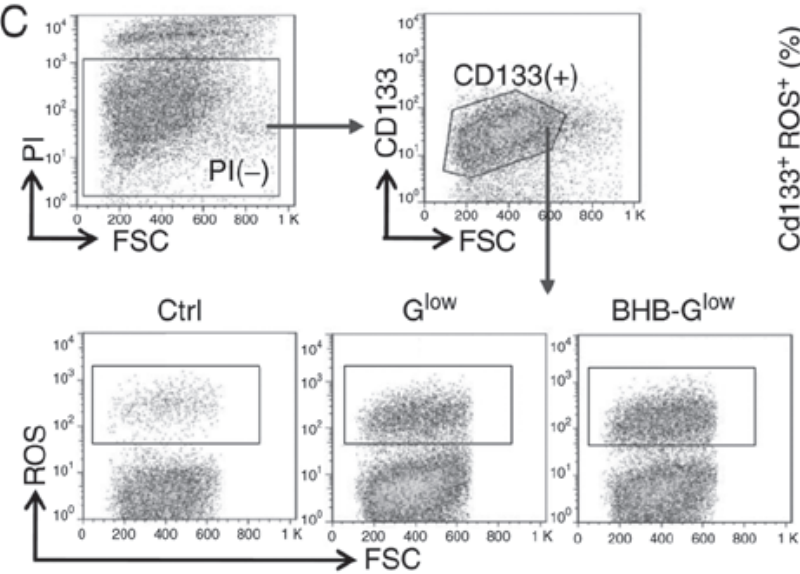
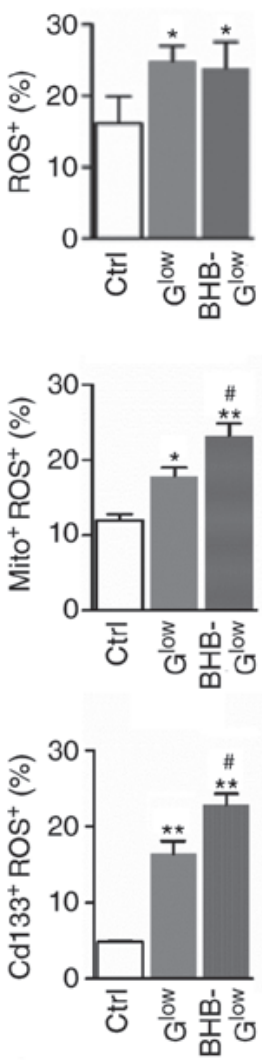
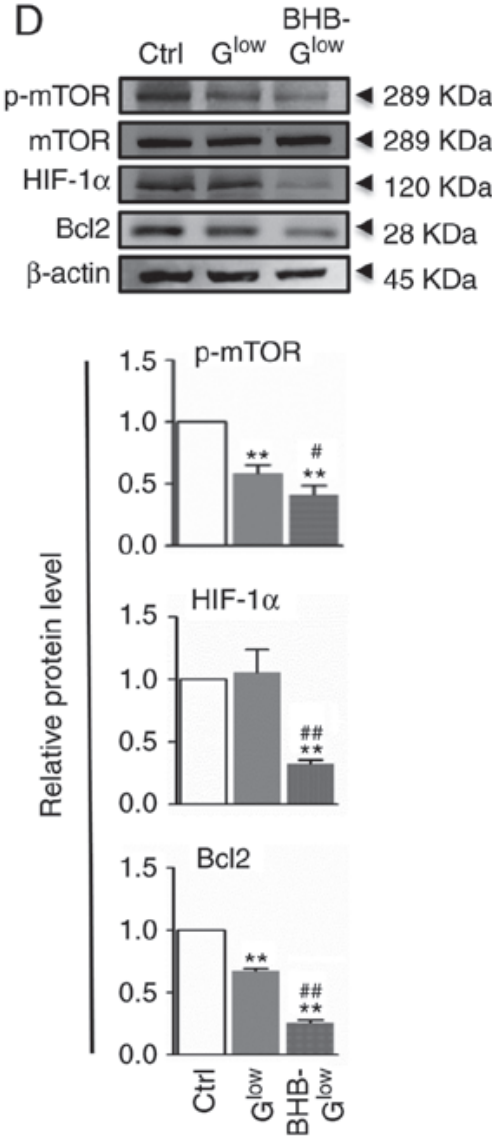

Figure 6. ROS production is increased in GSCs treated with BHB. (A) ROS production was evaluated using FACS in cells after staining with DCFH-DA and quantitatively compared.(B) Cells in (A) were stained with MitoTracker and DCFH-DA and analyzed by FACS. The MitoTracker ${ }^{+}$ROS ${ }^{+}$populations were quantitatively compared. (C) Cells in (A) were stained with anti-CD133 and DCFH-DA and analyzed by FACS. The CD133 ${ }^{+}$ROS ${ }^{+}$cells were quantitatively compared. (D) Cells in (A) were analyzed by western blotting for p-mTOR, mTOR, HIF-1 $\alpha$ and Bcl2, with $\beta$-actin used as the internal control. Data are presented as the mean \pm standard deviation. ${ }^{*} \mathrm{P}<0.05,{ }^{* *} \mathrm{P}<0.01$ vs. Ctrl; ${ }^{\#} \mathrm{P}<0.05,{ }^{\# \#} \mathrm{P}<0.01$ vs. $\mathrm{G}^{\text {low }}$. GSC, glioma stem-like cell; BHB, $\beta$-hydroxybutyrate; Ctrl, control medium with $25 \mathrm{mM}$ glucose; $\mathrm{G}^{\text {low }}$, medium with $2.5 \mathrm{mM}$ glucose; BHB-G ${ }^{\text {low }}, \mathrm{G}^{\text {low }}+\mathrm{BHB}$; ROS, reactive oxygen species; DCFH-DA, dichloro-dihydro-fluorescein diacetate; Mito, MitoTracker; p-, phospho; mTOR, mammalian target of rapamycin; FSC, forward scatter; PI, propidium iodide; HIF-1 $\alpha$, hypoxia-inducible factor $1 \alpha$; Bcl-2, B-cell lymphoma 2 .

treatment increased the expression of Sox 2 in NCH421k cells treated with BHB (Fig. 7D). Subsequently, metabolic changes in cells treated with NAC were determined. Intake of glucose was increased when ROS were scavenged in NCH421k cells cultured in BHB- $\mathrm{G}^{\text {low }}$ medium, as shown by the 2-NBDG assay results (Fig. 7E). TEM showed that the size of the mitochondria was increased in $\mathrm{NCH} 421 \mathrm{k}$ cells treated with NAC (Fig. 7F). These data suggest that ROS scavenging partly rescued the inhibitory effects of BHB in NCH421k cells.

\section{Discussion}

Abnormal metabolism, as represented by the Warburg effect, is one of the hallmarks of cancer and is critically involved in cancer initiation, malignant growth, metastasis and therapy resistance $(8,17)$. It has been reported that mutational and/or epigenetic modifications in cancer cells results in enhanced glycolysis even under aerobic conditions to meet the increased demands for energy, biosynthesis and gene expression regulation in cancer cells (5). Therefore, it is hypothesized, and to certain extent demonstrated, that cancer cells are dependent on glycolysis, and disruption of this glycolysis dependence could serve as potential anti-tumor therapy strategy (3). Ketone bodies, which are composed of acetoacetate, BHB, and acetone, are a degradation product of fatty acids produced in the liver and utilized by neuronal cells. While normal neuronal cells can oxidize ketone bodies in the mitochondria, glioma cells cannot, due to their metabolic reprogramming. Therefore, as a potentially effective therapy against glioma, the objective of a KD is to nourish normal neuronal cells with ketone bodies under glucose deprived conditions. In the brain, BHB is generated from oxidation of fatty acids or catabolism of amino acids in astrocytes, which can be metabolized in the mitochondria of all brain cell types although uptake across the blood brain barrier is a metabolic control point (30). Under glucose deprived conditions, cells will increase BHB usage as an alternative energy resource (31). However, glucose deprived glioma cells will starve, as they cannot not utilize 

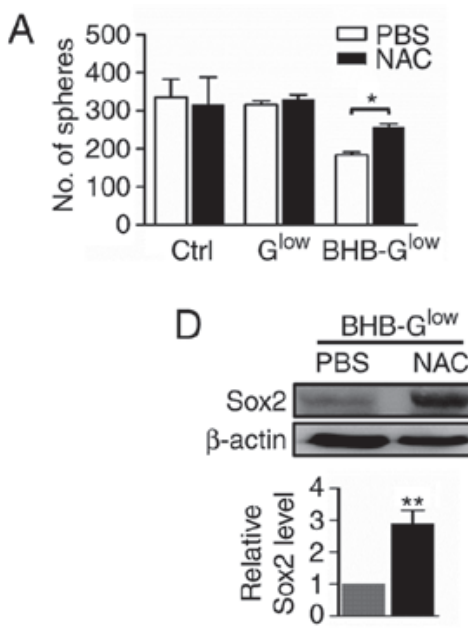
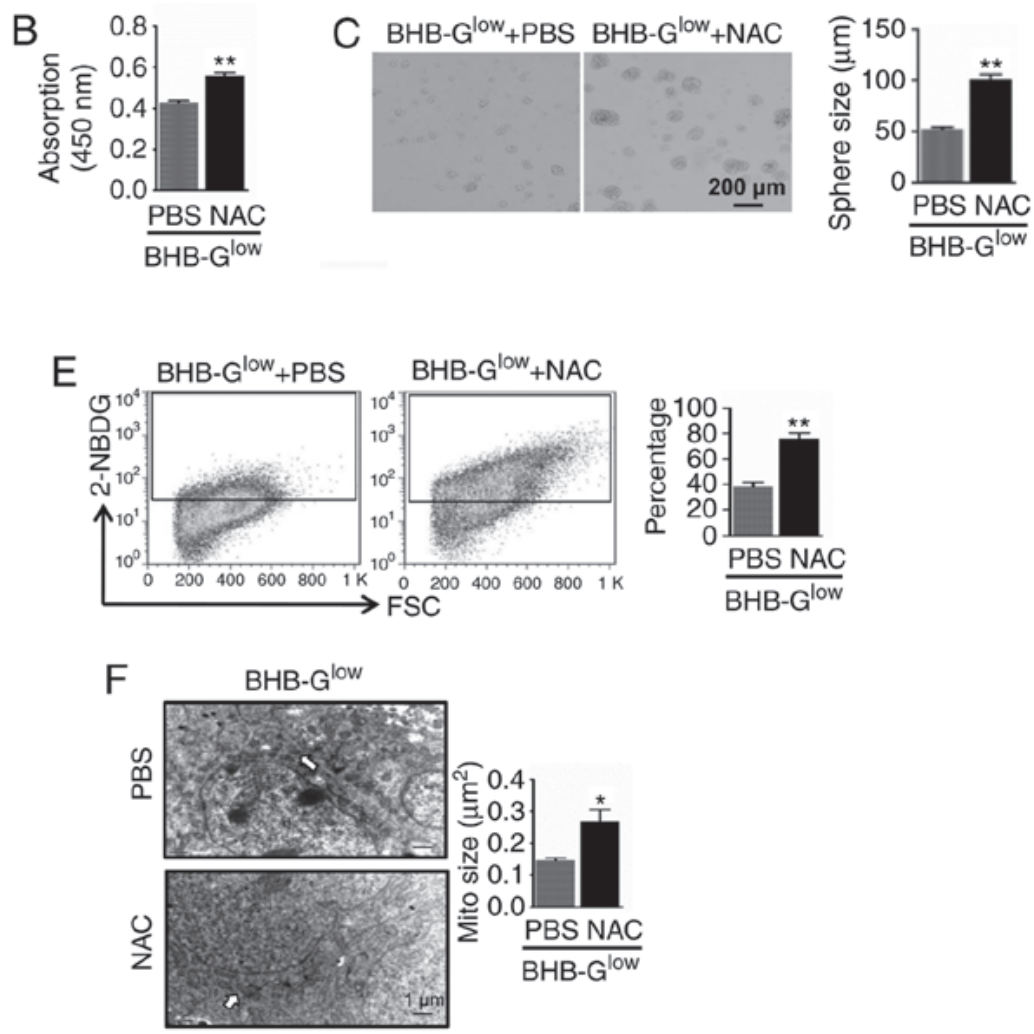

Figure 7. ROS scavenging rescues BHB-induced GSC inhibition. NCH421k cells were cultured in Ctrl, $\mathrm{G}^{\text {low }}$ or $10 \mathrm{mM}$ BHB-G ${ }^{\text {low }}$ medium for 7 days with or without 5 mM NAC. (A) Sphere formation and (B) cell viability were determined. (C) Tumor spheres were imaged, and the diameter of the spheres compared. Scale bar, $200 \mu \mathrm{m}$. (D) Expression of Sox 2 in cells in (C) was determined by western blotting and quantitatively compared. (E) Glucose transport in cells in (C) for 5 days was determined using 2-NBDG staining followed by FACS. (F) Cells in (C) for 5 days was imaged by transmission electron microscopy and the size of the mitochondria (white arrows) were quantitatively compared. Scale bar, $1 \mu \mathrm{m}$. Data are presented as the mean \pm standard deviation. ${ }^{*} \mathrm{P}<0.05,{ }^{* *} \mathrm{P}<0.01$ vs. Ctrl. GSC, glioma stem-like cell; BHB, $\beta$-hydroxybutyrate; Ctrl, control medium with 25 mM glucose; $\mathrm{G}^{\text {low }}$, medium with 2.5 mM glucose; BHB-G ${ }^{\text {low }}$, PBS $^{\text {low }}+$ BHB; ROS, reactive oxygen species; 2-NBDG, v2-[N-(7-nitrobenz-2-oxa-1,3-diaxol-4-yl) amino]-2-deoxyglucose; FSC, forward scatter; NAC, $\mathrm{N}$-acetylcysteine; Mito, MitoTracker.

ketone bodies. Glucose deprivation, a basic principle of a KD, is now considered as an adjuvant anti-glioma therapy based on in vitro and in vivo studies of its effectiveness $(10,12,32,33)$. However, the specific molecular mechanisms underlying the effectiveness of this approach against GBM, specifically GSCs, have remained elusive.

In the present study, the effects of a KD model on GSCs, specialized GBM cells with stem-like cell properties that have been shown to be responsible for drug resistance and tumor recurrence, were assessed. As BHB is the major component of ketone bodies under physiological ketosis, GSCs were cultured with BHB in low glucose medium. Based on clinical ketoacidosis and the blood-brain barrier, which restricts molecule exchange between the cerebral and peripheral compartments $(5,20)$, ketosis was mimicked using $2.5 \mathrm{mM}$ glucose and $<15 \mathrm{mM}$ BHB in the culture medium. The results showed that $\mathrm{BHB}$ supported the growth of normal mouse NSCs but inhibited the formation and growth of tumor spheres derived from GSCs. Additionally, BHB inhibited GSC proliferation and promoted GSC apoptosis under glucose deprived conditions, thus providing an explanation for the reduced sphere formation. The stemness of GSCs was also attenuated by BHB under the low glucose conditions. Together, these data provide further in vitro evidence supporting the therapeutic use of a KD for treatment of glioma; that is, normal NSCs and GSCs respond to a KD differently due to the intrinsic metabolic reprogramming in GSCs, and thus a KD can reduce GSC activity without significantly impairing normal NSC activity.

It is possible that BHB reduces GSC activity under glucose deprived conditions by restricting glycolysis-dependent metabolism $(20,34,35)$, a hallmark of GSCs (36). In the in vitro culture system, BHB reduced the expression and activity of Glut1 and several enzymes critical for glycolysis. This may result in an energy crisis in GSCs due to their dependence on glycolysis (37), as supported by the observed reduction in ATP production. Reduced ATP production hampers proliferation of cells and induces apoptosis in GSCs. Interestingly, inconsistent with the attenuation of glycolysis, lactate production was increased in BHB-treated cells. These findings suggest that BHB not only inhibits glycolysis, but also damages mitochondria in GSCs, and thus further demonstrate the anti-tumor effects of a KD (13). Damage to the mitochondria results in oxidative stress, which disturbs the intracellular balance between antioxidants and the extent of ROS generation, initiating a wide array of cellular responses via multiple signaling pathways, including the anti-oxidative enzymes SOD, CAT, and GSH (38). Consistent with this notion, ROS production was increased alongside the decreased activity and concentration of anti-oxidative enzymes in GSCs treated with BHB. 
Additionally, morphological changes were observed in the mitochondria, most notably a reduction in mitochondrial size, and the mitochondrial transmembrane potential was reduced. Antioxidant treatment with a ROS scavenger abrogated the inhibition of cell proliferation and self-renewal mediated by $\mathrm{BHB}$, further demonstrating that increased ROS production serves an important role in the BHB-induced inhibition of GSCs. GSCs presented higher levels of ROS compared with NSCs and scavenging ROS under glucose deprived conditions did not significantly affect proliferation (Fig. 7A), indicating that other elements in the microenvironment, not ROS, prolonged cell survival during glucose deprivation in GSCs.

The complete mechanism underlying BHB-induced GSC inhibition may be more complicated. ROS can directly regulate multiple signaling pathways such as the Akt-mTOR-HIF-1 $\alpha$ pathway, leading to changes in cell proliferation and apoptosis (39). BHB treatment reduced the levels of mTOR phosphorylation as well as the expression of HIF-1 $\alpha$ and $\mathrm{Bcl} 2$. Furthermore, previous studies have shown that $\mathrm{BHB}$ is an intrinsic histone deacetylase inhibitor, and thus regulates large-scale alterations in gene expression through histone modification, resulting in multiple cellular changes $(40,41)$. BHB-induced ROS production appeared to be greater in $\mathrm{CD} 133^{+}$GSCs compared with those negative for CD133, suggesting that a KD may target different GSC populations with differing efficacies. The pathogenesis of glioma is complicated, and involves numerous signaling pathways (7). Thus, additional studies on the effects of a KD on GSCs are warranted, given the critical roles of GSCs in the progression of glioma. In addition, the present study, the effect of a KD on GSCs in conditions of physiological ketosis in vitro were examined. However, the clinical significance is unknown based on the results of the present study. This is partly because the level of ketone bodies in the circulating blood, or 'ketosis' limits the concentration in the brain $(5,13)$. Under physiological conditions, the concentration of blood ketone bodies is inversely proportional to the concentration of blood glucose. Generally, cells including GSCs, are have sufficient glucose as an energy resource. If glucose supplies are limited, other metabolites, such as ketone bodies, serve as the energy source. The effective management of the balance between glucose and ketone bodies influences the therapeutic effects of the ketogenic diet in both cell lines and animal experiments $(10,42)$. One limiting factor in the use of a KD is treatment compliance, which is unsatisfactory, particularly in adults. Considering the adherence and ketogenic management in patients (43), in vivo studies are required to further optimize KD treatment for clinical use.

In conclusion, the results of the present study, together with those of published studies, support the hypothesis that GSCs have a unique metabolic profile that differs from that of normal NSCs and is highly dependent on glycolysis as an energy source. A KD which supplies ketone bodies combined with low glucose levels disturbs the metabolic balance in GSCs, leading to an energy crisis and thus, oxidative stress, which in turn reduces cell proliferation and stemness and even induces apoptosis. Therefore, by targeting GSCs, a KD may serve as an adjuvant therapy for treatment of patients with GBM.

\section{Acknowledgements}

The authors would like to thank Dr. Kewei Fang of Shenzhen Children's Hospital (Shenzhen, China) for his assistance with the ketogenic diet treatment protocol.

\section{Funding}

The present study was supported by grants from the National Natural Science Foundation (grant nos. 31671523 and 31101054), the Innovation Military Project of China and the First Affiliated Hospital Program (grant no. 2018QN-09).

\section{Availability of data and materials}

The datasets used and/or analyzed during the present study are available from the corresponding author on reasonable request.

\section{Authors' contributions}

$\mathrm{HH}$ and $\mathrm{ZF}$ designed the study. FW supervised the study. CJ, $\mathrm{YH}, \mathrm{CG}, \mathrm{LL}$ and BG performed the GSC in vitro experiments and analyzed the data. CJ, YR and XC performed the GSC in vivo experiments and analyzed the data. CJ, JL, MZ and SL analyzed the immunofluorescence, transmission electron microscopy and hematoxylin and eosin staining data. CJ, LL BG wrote the manuscript. All authors read and approved the final manuscript.

\section{Ethics approval and consent to participate}

The use of human samples was approved by the Ethics Committee of Xijing Hospital (Xi'an, China) and procedures were performed in accordance with the Declaration of Helsinki. Written informed consent was obtained from all patients involved.

\section{Patient consent for publication}

Not applicable.

\section{Competing interests}

The authors declare that they have no competing interests.

\section{References}

1. Poteet E, Choudhury GR, Winters A, Li W, Ryou MG, Liu R, Tang L, Ghorpade A, Wen Y, Yuan F, et al: Reversing the Warburg effect as a treatment for glioblastoma. J Biol Chem 288: 9153-9164, 2013.

2. Stupp R, Mason WP, van den Bent MJ, Weller M, Fisher B, Taphoorn MJ, Belanger K, Brandes AA, Marosi C, Bogdahn U, et al: Radiotherapy plus concomitant and adjuvant temozolomide for glioblastoma. N Engl J Med 352: 987-996, 2005.

3. Maurer GD, Brucker DP, Bähr O, Harter PN, Hattingen E, Walenta S, Mueller-Klieser W, Steinbach JP and Rieger J: Differential utilization of ketone bodies by neurons and glioma cell lines: A rationale for ketogenic diet as experimental glioma therapy. BMC Cancer 11: 315, 2011.

4. Vander Heiden MG, Cantley LC and Thompson CB: Understanding the Warburg effect: The metabolic requirements of cell proliferation. Science 324: 1029-1033, 2009. 
5. Seyfried TN, Flores R, Poff AM, D'Agostino DP and Mukherjee P: Metabolic therapy: A new paradigm for managing malignant brain cancer. Cancer Lett 356: 289-300, 2015.

6. Hoskin PJ, Sibtain A, Daley FM and Wilson GD: GLUT1 and CAIX as intrinsic markers of hypoxia in bladder cancer: Relationship with vascularity and proliferation as predictors of outcome of ARCON. Br J Cancer89: 1290-1297, 2003.

7. Ward PS and Thompson CB: Metabolic reprogramming: A cancer hallmark even warburg did not anticipate. Cancer Cell 21: 297-308, 2012.

8. Shen YA, Wang CY, Hsieh YT, Chen YJ and Wei YH: Metabolic reprogramming orchestrates cancer stem cell properties in nasopharyngeal carcinoma. Cell Cycle 14: 86-98, 2015.

9. Klement RJ, Bandyopadhyay PS, Champ CE and Walach $\mathrm{H}$ : Application of Bayesian evidence synthesis to modelling the effect of ketogenic therapy on survival of high grade glioma patients. Theor Biol Med Model 15: 12, 2018.

10. Augur ZM, Doyle CM, Li M, Mukherjee P and Seyfried TN: Nontoxic targeting of energy metabolism in preclinical VM-M3 experimental glioblastoma. Front Nutr 5: 91, 2018.

11. Champ CE, Palmer JD, Volek JS, Werner-Wasik M, Andrews DW, Evans JJ, Glass J, Kim L and Shi W: Targeting metabolism with a ketogenic diet during the treatment of glioblastoma multiforme. J Neurooncol 117: 125-131, 2014.

12. Martuscello RT, Vedam-Mai V, McCarthy DJ, Schmoll ME, Jundi MA, Louviere CD, Griffith BG, Skinner CL, Suslov O, Deleyrolle LP and Reynolds BA: A supplemented high-fat low-carbohydrate diet for the treatment of glioblastoma. Clin Cancer Res 22: 2482-2495, 2016.

13. Poff A, Koutnik AP, Egan KM, Sahebjam S, D'Agostino D and Kumar NB: Targeting the Warburg effect for cancer treatment: Ketogenic diets for management of glioma. Semin Cancer Biol 56: 135-148, 2017.

14. Ito K and Suda T: Metabolic requirements for the maintenance of self-renewing stem cells. Nat Rev Mol Cell Biol 15: 243-256, 2014

15. Sell S: On the stem cell origin of cancer. Am J Pathol 176 2584-2594, 2010.

16. Batlle E and Clevers H: Cancer stem cells revisited. Nat Med 23: 1124-1134, 2017

17. Folmes CD, Dzeja PP, Nelson TJ and Terzic A: Metabolic plasticity in stem cell homeostasis and differentiation. Cell Stem Cell 11: 596-606, 2012.

18. Nakano I: Therapeutic potential of targeting glucose metabolism in glioma stem cells. Expert Opin Ther Targets 18: 1233-1236, 2014.

19. Cairns RA, Harris IS and Mak TW: Regulation of cancer cell metabolism. Nat Rev Cancer 11: 85-95, 2011.

20. Flavahan WA, Wu Q, Hitomi M, Rahim N, Kim Y, Sloan AE, Weil RJ, Nakano I, Sarkaria JN, Stringer BW, et al: Brain tumor initiating cells adapt to restricted nutrition through preferential glucose uptake. Nat Neurosci 16: 1373-1382, 2013.

21. Agnihotri S and Zadeh G: Metabolic reprogramming in glioblastoma: The influence of cancer metabolism on epigenetics and unanswered questions. Neuro Oncol 18: 160-172, 2016.

22. Zhou Y, Zhou Y, Shingu T, Feng L, Chen Z, Ogasawara M, Keating MJ, Kondo S and Huang P: Metabolic alterations in highly tumorigenic glioblastoma cells: Preference for hypoxia and high dependency on glycolysis. J Biol Chem 286 : 32843-32853, 2011.

23. Campos B, Wan F, Farhadi M, Ernst A, Zeppernick F Tagscherer KE, Ahmadi R, Lohr J, Dictus C, Gdynia G, et al: Differentiation therapy exerts antitumor effects on stem-like glioma cells. Clin Cancer Res 16: 2715-2728, 2010.

24. Gao F, Zhang YF, Zhang ZP, Fu LA, Cao XL, Zhang YZ, Guo CJ Yan XC, Yang QC, Hu YY, et al: miR-342-5p regulates neural stem cell proliferation and differentiation downstream to Notch signaling in mice. Stem Cell Reports 8: 1032-1045, 2017.

25. Hu YY, Fu LA, Li SZ, Chen Y, Li JC, Han J, Liang L, Li L, Ji CC, Zheng MH and Han H: Hif- $1 \alpha$ and Hif- $2 \alpha$ differentially regulate Notch signaling through competitive interaction with the intracellular domain of Notch receptors in glioma stem cells. Cancer Lett 349: 67-76, 2014.
26. Ilkhanizadeh $\mathrm{S}$ and Weiss WA: Starvation favors glioma stem cells. Nat Neurosci 16: 1359-1361, 2013.

27. Bassoy EY, Kasahara A, Chiusolo V, Jacquemin G, Boydell E, Zamorano S, Riccadonna C, Pellegatta S, Hulo N, Dutoit V, et al: ER-mitochondria contacts control surface glycan expression and sensitivity to killer lymphocytes in glioma stem-like cells. EMBO J 36: 1493-1512, 2017

28. Halabe Bucay A: The biological significance of cancer: Mitochondria as a cause of cancer and the inhibition of glycolysis with citrate as a cancer treatment. Med Hypotheses 69: 826-828, 2007.

29. Mischel PS: HOT Models in flux: Mitochondrial glucose oxidation fuels glioblastoma growth. Cell Metab 15: 789-790, 2012.

30. Bernini A, Masoodi M, Solari D, Miroz JP, Carteron L, Christinat N, Morelli P, Beaumont M, Abed-Maillard S, Hartweg M, et al: Modulation of cerebral ketone metabolism following traumatic brain injury in humans. J Cereb Blood Flow Metab: October 24, 2018 (Epub ahead of print).

31. Fusco S, Leone L, Barbati SA, Samengo D, Piacentini R, Maulucci G, Toietta G, Spinelli M, McBurney M, Pani G and Grassi C: A CREB-Sirt1-Hes1 circuitry mediates neural stem cell response to glucose availability. Cell Rep 14: 1195-1205, 2016.

32. Rieger J, Bähr O, Maurer GD, Hattingen E, Franz K, Brucker D, Walenta S, Kämmerer U, Coy JF, Weller M and Steinbach JP: ERGO: A pilot study of ketogenic diet in recurrent glioblastoma. Int J Oncol 44: 1843-1852, 2014.

33. Woolf EC, Curley KL, Liu Q, Turner GH, Charlton JA, Preul MC and Scheck AC: The ketogenic diet alters the hypoxic response and affects expression of proteins associated with angiogenesis, invasive potential and vascular permeability in a mouse glioma model. PLoS One 10: e130357, 2015.

34. Klement RJ and Champ CE: Calories, carbohydrates, and cancer therapy with radiation: Exploiting the five R's through dietary manipulation. Cancer Metastasis Rev 33: 217-229, 2014.

35. Venneti $\mathrm{S}$ and Thompson CB: Metabolic modulation of epigenetics in gliomas. Brain Pathol 23: 217-221, 2013.

36. Singer E, Judkins J, Salomonis N, Matlaf L, Soteropoulos P, McAllister S and Soroceanu L: Reactive oxygen species-mediated therapeutic response and resistance in glioblastoma. Cell Death Dis 6: e1601, 2015.

37. Fu X, Chin RM, Vergnes L, Hwang H, Deng G, Xing Y, Pai MY, Li S, Ta L, Fazlollahi F, et al: 2-Hydroxyglutarate Inhibits ATP Synthase and mTOR Signaling. Cell Metab 22: 508-515, 2015.

38. Sharma PK, Bhardwaj R, Dwarakanath BS and Varshney R: Metabolic oxidative stress induced by a combination of 2-DG and 6-AN enhances radiation damage selectively in malignant cells via non-coordinated expression of antioxidant enzymes. Cancer Lett 295: 154-166, 2010.

39. Zhao Y, Hu X, Liu Y, Dong S, Wen Z, He W, Zhang S, Huang Q and Shi M: ROS signaling under metabolic stress: Cross-talk between AMPK and AKT pathway. Mol Cancer 16: 79, 2017.

40. Shimazu T, Hirschey MD, Newman J, He W, Shirakawa K, Le Moan N, Grueter CA, Lim H, Saunders LR, Stevens RD, et al: Suppression of oxidative stress by $\beta$-hydroxybutyrate, an endogenous histone deacetylase inhibitor. Science 339: 211-214, 2013.

41. Benjamin JS, Pilarowski GO, Carosso GA, Zhang L, Huso DL, Goff LA, Vernon HJ, Hansen KD and Bjornsson HT: A ketogenic diet rescues hippocampal memory defects in a mouse model of Kabuki syndrome. Proc Natl Acad Sci USA 114: 125-130, 2017

42. Poff AM, Ari C, Arnold P, Seyfried TN and D'Agostino DP: Ketone supplementation decreases tumor cell viability and prolongs survival of mice with metastatic cancer. Int J Cancer 135: 1711-1720, 2014.

43. Chung H and Park YK: Rationale, feasibility and acceptability of ketogenic diet for cancer treatment. J Cancer Prev 22: 127-134, 2017. 